\title{
Analysis of the Contributions of Bulgarian Academy of Sciences in Development and Production of the Industrial Robots
}

\author{
Vassil Sgurev \\ ${ }^{I}$ Institute of Information and Communication Technologies, Sofia, Bulgaria \\ Emails:vvsgurev@gmail.com
}

\begin{abstract}
The present work describes the significant achievements of this institute, and of BAS in general in research, development, testing, production, elaboration of operative documentation, and direct - without intermediaries - implementation of robots and control devices to them in serial production in two plants in Stara Zagora - the National and Industrial Stara Zagora Plant for Robotics "Beroe" and for Disk Storage Devices. These are the high-tech sophisticated welding robots RB-250, painting, and training robots, and point-to-point robots. These achievements enabled the Bulgarian machine-building industry to take a leading role in robotics in the then eastern block of countries and beyond. The significant role of the Institute of Technical Cybernetics and Robotics at BAS for the development of industrial controllers and controllers for robots that meet extremely difficult industrial conditions and requirements is emphasized. The special role of the Institute's Experimental Base was emphasized, which became a kind of "technopark" for the rapid implementation of scientific achievements. The unified coordinated activity of the academic institute and the two economic organizations from the region of Stara Zagora is highlighted, which makes them a kind of cluster of robotics. It is emphasized that the positive experience described can also be used in today's market economy.
\end{abstract}

Keywords: Contributions, Bulgarian Academy of Sciences, Development and Implementation, Industrial Production of Robots.

\section{Analysis of the Contributions of BAS in Development and Production of the Industrial Robots}

In the founding document of November 30, 1978, establishing the Institute of Technical Cybernetics and Robotics (ITCR) at the Bulgarian Academy of Sciences 
(BAS), one of its two main objectives is formulated as follows: experimental and applied research in the fields related to with cybernetic systems for managing technical processes and sites; research and implementation in the field of robots and their accelerated application in the national economy, pilot production and training, preparation and postgraduate qualification of specialists in these fields.

To solve these major tasks of ITCR, an industrial site of 140 acres was transferred to Sofia, together with an existing plant, to be used as an Experimental Base of the Institute for experimentation, prototyping, pilot production, production of operative documentation for the batch production of the created at the Institute robots. In this way, a united complex "Academic Institute - Experimental Base" is created, in which the cycle "research - implementation of robots" is completely closed. Substantial investment funds and staffing levels have also been allocated for staffing this activity. This creates opportunities that have only the best research units of the Bulgarian industry of the time. A unique innovation model is being formed at BAS, which in practice proves to be very successful.

Prior to the creation of ITCR in its predecessor, the Institute of Technical Cybernetics (ITC) at BAS, the experience was gained in creating highly reliable computing structures capable of operating in harsh industrial environments. At the Institute of Mathematics (IM) at BAS, there are developments in the application of AI elements in various control structures. This experience of both academic institutes has been used in the development of different robots.

On the part of mechanical engineering as the basic enterprises for serial production of the industrial robots and control devices thereto, the corresponding National and Industrial Stara Zagora Plant for Robotics (NISPR) "Beroe" and for Disk Storage Devices (DSD) in Stara Zagora have been designated.

In the ITCR creation, the individual sections and ancillary units were formed in three separate directions, almost equal in number, namely technical cybernetics, robotic systems, and DIEP (Development, Implementation, and Experimental Production).

On the direction of Assoc. Prof. Dr. Bogdan Stoyanov, the DIEP direction is the main burden for the design and construction of the electromechanical part of robots and their systems. The department includes the following units: ElectroStructural Department; Mechanical Engineering Department; Technical Documentation and Archives, including Norms Control and Standards; Breeding Base; Electrical Assembly Workshop; Mechanical Workshop; Automated Circuit Board Design. With the involvement of the managerial and support staff, about 140 people are enrolled in the DIEP, one-third of the Institute's staff then. Together with the Experimental Base, the DIEP direction is a highly skilled force capable of moving and implementing significant and important projects in the field of robotics, personal computers, and cyber systems in the country. There are no such complex possibilities for practical implementation in BAS until the changes in 1990, neither after that nor now. 
Specialists from the Central Institute of Computer Engineering (CICE) and the metal-cutting industry have been accepted into the units with an applied orientation. In robotics, this is dictated by the similarity between machine tools and robots, resulting from:

- Similar motion geometry - kinematics, speed, translation, and rotation in start-stop mode;

- Similar element base;

- Actuators, clutches, brakes, bearings, kinematic transmission elements (ball-screw pairs), etc.;

- Similar 'drive, stop and feedback' system, as well as similar clearance mechanisms and clearances;

- Use of similar technological solutions in chemical-thermal and surface treatment.

The main contingent of specialists involved in the electromechanical part of robotics are from the Plant for Machine Tools (PMT)-Sofia and from the Institute for Research and Development on Machining by Special and Aggregate Machines (IRD MSAM). They form the core through which the corresponding developments are realized.

The first very responsible task of ITCR-BAS in robotics is the elaboration of a microprocessor control device for hydraulic robots of the VERSATRAN-AMF type. The manufacturing license for the mechanical actuator (the manipulators) was purchased from the "Beroe" NISPR but without the controllers considered as an embargo product. ITCR has been tasked with developing such controllers that are not inferior to the original company products.

Until then, in Bulgaria, there has been no experience in creating such a class of controllers that can operate in too harsh production conditions - polluted air, vibrations, and electric shocks due to the start-stop modes of powerful motors in metalworking and other machines. Controllers for welding devices and cranes must have a high degree of ISO protection, they must have maximum seals and cooling with counter-flows from the outside and inside air.

It was decided to fit the electronic circuit boards into the motherboard cassette, thus reducing some of the cables. It is also necessary to develop a power supply unit with filters and stabilizers, as well as a control operator's panel plus a training panel. This requires the selection and construction of a suitable radiator filter to cool the recycled stream of filtered air, which is complex and technologically difficult to manufacture, as well as a number of sealing elements.

Such versatile and mutually contradictory requirements lead to the creation of effective well-researched and designed controllers, which are very different from the existing controllers in metalworking and computer engineering.

Training specialists to accomplish such tasks is too difficult a task in itself. It is implemented "on the go", according to accelerated programs. 
In the end, the task of developing specialized robotics controllers was successfully accomplished by ITCR and the Bulgarian industry received verified operative documentation for their serial production - in turn successfully accomplished for several years.

The robotic complex RB-250 for arc welding in those years was the most sophisticated and high-tech product in Bulgarian robotics, and probably also in Bulgarian mechanical engineering. Its welding part was realized as a joint development between ITCR and the Paton Institute of the Academy of Sciences of the Ukrainian SSR. Most of this activity was carried out within the framework of the Joint Interrobobosvarka Laboratory located in Sofia between the ITCR and the Paton Institute.

The RB-250 is designed to implement the continuous path welding scheme. This manufacturing operation is extremely complex; with unevenness between the two welded parts, a solid high-quality weld is required. Such a seam is available only to highly skilled and costly welders. Thanks to the numerous sensors fitted, the successfully selected feedbacks, and the sophisticated software, robotic welding resembles, and in some cases outperforms, the work of a highly-skilled welder somewhere with greater, but somewhere with less success. The temperature of the weld is judged by the shape and volume of the molten metal bath. The binding of the information from the sensors with the feedback and the creation of optimal control effects for welding is performed by carefully selected algorithms in the specialized multiprocessor system. It was developed at ITCR, but it is produced at the DSD Stara Zagora according to the operative documentation from the institute. The robotic complex RB-250 includes a source for welding current, a water-cooled burner with devices for cleaning the burner, and trimming the welding wire after each duty cycle, as well as the unit and hoses for cooling the burner and water recirculation.

Soft motors, incremental sensors are used for incremental feedback for position, speed and acceleration, as well as for positioning stops. The transformation of rotational motion into translational is carried out by means of imported ball-pair couplings (LEDs). After much discussion, LEDs with rolled screws with a limited cumulative system error are accepted. The achieved positioning up to $\pm 0.25 \mathrm{~mm}$ and repeatability of $\pm 0.15 \mathrm{~mm}$ is too high precision for electromechanical devices. In order to ensure the reliable operation of the complex, specific devices are also being developed such as safety connectors against breaking, pneumatic cylinders, and counterweights for counterbalancing and facilitating the vertical movement of the robot; telescopic protection of the guides against dust and splashes from arc welding.

All mechanical and electro-mechanical details of the complex have been developed, constructed, and worked up to functional drawings in ITCR and its Experimental Base. This includes the selection of materials and tooling and the prototype design. A complete set of functional drawings, which barely fit into an eight-seater minibus, has been handed over to the NISPR "Beroe". 
By contract, the "Beroe" NISPR produced 15 RB-250 robotic complexes for the needs of the Soviet Military-Industrial Complex (MIC). They have been shipped to the Ukrainian SSR, but our institute specialists are not allowed to install or operate them. According to unconfirmed data, they are used for automatic welding of products in Soviet naval shipbuilding. After a while, we were informed that the complexes were operating normally and that the buyer had no claims against the manufacturer. This was good for the ITCR development teams, the "Beroe" NISPR, and the Disk Storage Devices (DSD) - Stara Zagora.

The success of RB-250 is also aided by several original copyright certificates and patents embedded in this welding complex - the mechanics, control, and filing of the welding wire. Of these, the most impressive is protected by several patents body-feeder device, developed by a team led by Assoc. Prof. David Samokovliyski. It was also manufactured as a standalone product from our Experimental Base and it received relatively wide distribution in our country and abroad - in the UK, USA, a number of Latin American and Western European countries. Assoc. Prof. D. Samokovliyski travelled constantly and with good advertising and demonstrations he helped a lot for the commercial success of the device. The institute received a letter from the management of a London shipyard stating the good work of our feeder.

It is fair to note that the software sections of the Institute worked continuously to improve the software of the robotic complex RB-250, as well as of other robots developed in ITCR.

Two other classes of industrial robots - point-to-point and painting automation robots [1] have been developed using well-known and well-presented kinematic schemes in ITCR. In mechanical engineering, and not only there are many operations where certain parts need to be moved from one place to another. Making such moves is relatively easy with point-to-point robots. In a simple kinematic scheme, it was necessary to create a ruling controller that would meet their conditions during the industrial operation of the robot. The team, led by Assoc. Prof. Georgi Nachev, successfully accomplished this task. Thus, the serial production and replication of these robots was ensured at home and abroad. But that was already the task of the "Beroe" NISPR and the DSD in Stara Zagora

While these robots had simple software, things were different in the class of painting robots. There was a need for sophisticated and significant software, which had to replicate the movements of an experienced specialist in painting certain areas. Software professionals successfully dealt with this challenge as well, and the two Stara Zagora plants included in their sales nomenclature the commercially produced paint robots.

Another class of successfully developed and commercially produced robots, namely the ROBCO training robots, should be noted. Initially, they did not fit into the plans of the institute and they were imposed mainly due to the initiative of Assoc. Prof. Nedko Shivarov. These robots were for training purposes, small and inexpensive, with relatively simple mechanics, most commonly controlled through 
the 8-bit IMCO-2 PRAVETS-82 developed in ITCR [2], [3], [4]. The tiny ROBCO robots, in various variants and for different purposes, quickly gained popularity and were widely used, especially in schools and universities. The corresponding funding from the Ministry of Education and Science contributed to this. They were produced relatively easily by the Institute's Experimental Base. Of interest is the fact that they can still be found in some schools today.

The significant results in the development and production of industrial robots in our country are due in no small part to the direct - without intermediaries connections and coordination in the work of the ITCR and the two Stara Zagora business organizations. It could not have been otherwise - the deadlines were very tight and the responsibilities too great. The production of these high-tech products based on new breakthrough technologies was strictly monitored and controlled by the relevant state and party authorities.

It should be noted here the huge role of the director of the institute Assoc. Prof. Angel Angelov, who - thanks to his considerable connections in the industry and his position as a Deputy Chairman of the State Committee for Science and Technical Progress (SCSTP) - provided invaluable support to the Robotics Division. At the end of 1982 he was appointed Bulgarian Ambassador to Japan and then he left for Tokyo. His directing functions were performed by Prof. Dr. Sc. Vassil Sgurev by the end of 1987. From 1988 to 1991, he was chairman of the Board of Directors of the Beroe NISPR, and this circumstance also facilitated the close links and coordination between the two organizations. The activity continued at an intense pace and the results were not delayed.

It should not give the impression that the development of robots and robotic systems comes down to the design of electromechanical parts and devices. The development of appropriate high-performance software that is of high quality and "foolproof" and has reliable security against human-robot physical collisions is a significant part of this activity. A considerable number of highly qualified researchers and programmers were working on these problems in the developed sections of the Institute, whose task was to create exactly such software. The largest activity in this area was carried out in the section "Robot Management". It created a multi-level and multi-module software system for robot control - REX, incorporating the following modules in its structure: the operative environment; an incremental compiler that implements a step-by-step user program, whereby the compiler program is initially adjusted according to the current situation. The planning system has two basic blocks, one of which performs the planning, and the other detects the differences between the model created on a hierarchical basis and the true state of the operative environment. The new information is introduced via an orthogonal method. The objects of the technological process have a 3D representation. The software of the technological object thus constructed, containing AI elements, is taken as the basis for the training of industrial robots. 
In the Cybernetic Systems' Section (headed by Prof. Dr. Tech. Sciences Vassil Sgurev), considerable research has been carried out on the creation of various diagnostic and intelligent systems. For this purpose, the problem-independent expert system DIGS was elaborated [5]. It incorporates tools for rapid and efficient adjustment of diagnostic activity from one technical system to another. DIGS has built-in methods for diagnosing an industrial robot and for planning its activity. A network model has been elaborated that reflects the relationships between the different subsystems so that the user himself/herself can generate different hypotheses and he/she can introduce them into the expert system. The language "Prolog" is actively used.

The System Research Section (headed by Assoc. Prof. Dr. Ivan Popchev) directs a significant part of its efforts to construct the reliability of various systems, including robotic systems. The ways in which knowledge is presented and evaluated are explored. Appropriate software and decision support systems have been proposed.

Two international ROBCON conferences were held at the initiative and organization of ITCR - in 1985 and in 1987. Robotics management was also actively discussed at the AIMSA series of international conferences on artificial intelligence, held consecutively in 1984, 1986, 1988, 1990, 1992, and 1994. It was also initiated and organized by ITCR.

The activities of the ICTR in the field of robotics have been monitored and actively supported by the Presidium of BAS, which has discussed it at several of its meetings and measures were taken to support it. The chairman of the academy, Academician Angel Balevski, kept a direct account of the work of the institute and he actively assisted in solving its problems before high authorities.

The joint work of ITCR with the "Beroe" NISPR has gradually become a single, synchronously working organizational mechanism. Several ITCR teams worked simultaneously in the Stara Zagora plants, which required the institute to rent accommodation for its long-term seconded associates. ITCR took an active part in the establishment and construction of the Institute of Robotics in Stara Zagora, which was essentially its affiliate - its associates were trained through joint work with the Sofia developers. The staff of ITCR actively participated in the selection and purchase of up-to-date equipment and new technologies for the needs of the Beroe NISPR. One of the first flexible automated production systems (FAPS) was installed in the same plant, which was then on the cutting edge of machine-building technologies. This FAPS was solemnly opened on October 5, 1983, in the presence of responsible state officials. Scientists and specialists from ITCR and the management of BAS were invited and participated.

NISPR "Beroe" also purchased a license for the production of ball-screw pairs, which was carried out with the active participation of ITCR specialists. Stara Zagora has gradually become an attractive innovation ground that the Bulgarian government has used to demonstrate the high-tech capabilities of our country. There was criticism 
from other regions of the country at the Centre in Sofia that it was investing too much in innovative development and production in the Stara Zagora region.

Other developments are known - for example, in QAM (Quality, Ambition, Motivation), Plovdiv, manipulators were developed, elaborated at HMEEI (Higher Mechanical and Electrical Engineering Institute), Sofia. But this activity was far more modest in scope than the described one in this article. At that time there were other attempts at BAS to develop robotic devices, but they remained only as experimental specimens in the absence of industrial production.

In conclusion, the following may be noted:

- The active and decisive participation of BAS at the end of the last century through its Institute of Technical Cybernetics and Robotics in the elaboration, development, and serial production of robotic systems is a clear example of a modern fast transition to high-tech products and systems for the country's needs and for export. In this area, the Bulgarian machine-building got ahead of the positions in the eastern block of countries then. And not only there.

- Of particular importance is the role of ITCR-BAS in the elaboration of industrial controllers and controllers for robots and robotic systems to meet the extremely demanding industrial conditions and requirements. The lack of experience has been overcome quickly and our country was able to develop and produce such scarce embargo products.

- The accelerated construction of a single complex by an academic institute with a well-equipped Experimental Base - essentially a Technopark - has proven to be a profitable strategy that is now being successfully used in many countries. This allowed testing, trial production, production of operative documentation, and a direct implementation without intermediaries in the industry and with significant benefits for the state. As well as a too fast payback.

- The existence of a well-thought-out and effective institutional social policy has enabled the implementation and the retention of highly qualified scientists and specialists in ITCR and its Experimental Base.

- The described approach allowed for the construction of a suitable and largely unified infrastructure of high-tech plants and an academic institute with an Experimental Base (in the modern "cluster" terminology), which was able to develop and produce high-tech products with increasing complexity in the nomenclature of mechanical engineering, industrial electronics, and robotics.

After the changes in Bulgaria in 1990, the elaboration and the serial production of industrial robots and robotic systems in Bulgaria were gradually discontinued. At the same time, several small companies appeared in the town of Stara Zagora and other regions of our country. They continued the traditions of Bulgarian robotics from the 70's and 80's of the last century. 
From any point of view, to look at the efforts of BAS to research, elaborate, and implement in the serial production of robots, it cannot be rejected that these efforts were successful and decisive for the development of the Bulgarian economy. The experience gained in this area of science can also be used to some extent nowadays when the creation and production of high-tech products - and robots and robotic systems are just such a class of products and systems - is becoming a national goal for many governments and countries. Moreover, it is obvious that such a national goal must be a leader also in our country.

\section{References}

1. Hunt, D.V.: Industrial Robotics Handbook. Industrial Press, Inc. (1983).

2. Vachkov, P., K. Boyanov, K. Yanev, Perspectives on the Development of Personal Computers in the 'Microprocessor Systems' Economic Union. In: Proc. of PERSCOM'87, vol. 1, pp. 51-63, (1987), (in Bulgarian).

3. Angelov, A., Y. Kisiyov, N. Iliev, Instrumental systems and computers. In: Proc. of PERSCOM'87, vol. 1, pp. 107-111 (1987), (in Bulgarian).

4. Sgurev, V., The state-of-the-art, the development and the perspectives of the personal computers of the 'Pravets' family. In: Proc. of PERSCOM'85, vol. 1, 1985, (in Bulgarian).

5. Agre, G., Sgurev, V., Dochev, D., Dichev, Ch., Markov, Z.: An implementation of the expert system DIGS for diagnostics. Computers and Artificial Intelligence 4(6), 495-502, (1985). 